\title{
Green Design and Recycling Systems for Solving the Dilemma of Disposable Chopsticks Waste Caused by Online Food Delivery: A Review
}

\author{
Yudong Fu and Jiangang Zhu* \\ With the rapid advancement of online food delivery and takeaway markets, \\ the use of disposable chopsticks has exponentially increased in China, \\ resulting in the reduction of forest resources and the generation of large \\ amounts of chopstick waste. This review summarizes a variety of methods \\ to produce eco-friendly chopsticks with the aim of enabling a second life \\ cycle for chopstick waste. The related practical recycling systems and \\ policies based on government experience in developed regions are \\ proposed. Furthermore, perspectives with regard to the future \\ development of waste chopsticks recycling systems are also discussed.
}

Keywords: Disposable chopsticks; Food delivery tableware; Green design; Recycling systems; Recycling strategies

Contact information: College of Furnishings and Industrial Design, Nanjing Forestry University, Nanjing 210037 PR China; *Corresponding author: austin_zhu@njfu.edu.cn

\section{INTRODUCTION}

Chopsticks, as traditional kitchen and eating utensils, are widely used in China and some East Asian countries because of their convenience and cheapness (Shih et al. 2012; Chen et al. 2017). In China, both wood and bamboo provide main sources of raw materials for the production of disposable chopsticks (DCs). Specifically, the raw materials of wooden DCs mainly come from fast-growing wood, e.g., poplar, birch, and pine in Northeast China, while the producing areas of bamboo DCs are concentrated in southern China, e.g., Hunan, Jiangxi and Fujian Province, with moso bamboo as the main material (Kissen 2017). Influenced by traditional culture and historical customs, Chinese prefer to use all kinds of wood products in daily life, and wooden chopsticks are no exception (Faraca et al. 2019), which is one of the reasons why wooden chopsticks account for half of the chopsticks market in China. However, the situation of China's forest resources is worrisome. The total forest area of China ranks among the top in the world, with approximately 221 million hectares, but per the capita forest area is only 0.145 hectares, less than a quarter of the per capita of the world. Although China's forest coverage rate has risen to $23.04 \%$ in 2020 , it is still far below the world's forest coverage rate of around $32 \%$. On the other hand, bamboo resources are relatively abundant in China, covering an area of approximately $520 \mathrm{hm}^{2}$, accounting for $1 / 4$ of the total area of the bamboo forests in the world, which makes it an ideal candidate to substitute for wood. Nevertheless, chopsticks are also one of the major sources of waste in China due to their short life cycle. Except for some expensive rosewood chopsticks that are used for collection and gifting, which can be stored for a long time (Liu et al. 2020), most chopsticks need to be replaced after 3 months to 6 months, considering health issues. The life cycle of DCs is much shorter, as they tend to be thrown into the trash after being used for only approximately 30 minutes, which poses 
a huge threat to natural resources and environmental protection efforts (Blanca-Alcubilla et al. 2020). Even worse, the online takeaway market in China has rapidly developed, which has led to a surge in the use of DCs and generated $323 \mathrm{kt}$ of tableware and packaging waste in 2018. It is estimated that a third of DCs consumption in China comes from the food delivery industry (Molina-Besch 2020; Zhou et al. 2020). Usually, the primary endof-life option for DCs is landfill and incineration (Xu et al. 2020). However, DCs may be equally well suited for various recycling options on account of its other inherent material characteristics (European Environment Agency 2016). Therefore, the recycling of DCs is urgently proposed to meet the ever-growing demands for a circular economy.

In this review, the authors report various novel approaches to handle the increasingly serious problem caused by chopstick waste. Additional attention is paid to the green designing of DCs, along with the recycling and reuse of chopstick waste. The green design aims to optimize the processing technique, seek eco-friendly alternative materials to improve the life cycle of DCs, and reduce the consumption of wood and bamboo resources. In addition, this work presents a state-of-the art overview on the recycling and utilization of chopstick waste, including the preparation of chemical raw materials, the developing of novel composite materials, the processing of wood products, etc. To put this topic in context, several disposable tableware recycling systems based on the specific situation in China are presented, with the garbage recycling situation and policies in different regions of China also being included. Finally, the primary issues that will be improved in the near future concerning chopstick recycling are shown.

\section{EMERGING CHALLENGE: TAKEAWAY TABLEWARE WASTE}

In China, the progress of e-commerce and changing lifestyles are reshaping fastdeveloping online food delivery service platforms, e.g., Meituan and Ele.me. The unique "Internet + catering" online-to-offline delivery mode is consistent with the modern fastpaced lifestyle and is favored by most young people. It is estimated that the number of online takeaway platform users in China has increased from 60 million in 2011 to 416 million in 2019 (Zhou et al. 2020). As shown in Fig. 1, the online food delivery business provided 5.57 billion orders to approximately 400 million users in 2017, and it showed a continual rising tendency, doubling in 2018 and rising to 17.1 billion in 2020 (Ju, 2021; $\mathrm{Li}$, 2018). Furthermore, the proportion of online food delivery turnover in China's total catering industry turnover rose from 1.4\% in 2015 to $10.6 \%$ in 2018 (Osens 2021; China Cuisine Association, 2018; Meituan Research Institute, 2018). In summary, the prosperity of the online food delivery industry has greatly boosted the consumption of DCs, and it has also brought emerging challenges to the natural environment and human health.

Statistically, the annual output of DCs in China is about 80 billion pairs (Zhongshang Industry Research Institute, 2018), of which around 35 billion pairs will be exported to Japan, Korea, and other regions (Fig. 2). The remaining 45 billion pairs of chopsticks are used for domestic consumption, of which about one-third are utilized in the food delivery industry, and the quantity is consistent with the annual takeaway orders. The production and processing of DCs are the most typical economic development model in developing countries, i.e., resource export-oriented productions are carried out to develop the economy. From 2017 to 2020, the average annual export of wooden DCs and bamboo DCs were 11.6 billion and 23.2 billion respectively (General Administration of Customs, 2020). The overexploitation of resources finally brings about the destruction of the 
environment. It takes a 30-year-old poplar tree to process 5000 pairs of wooden DCs. The production of DCs in China consumes more than 100 acres of forest every day, which is a total of 36000 acres in one year. Furthermore, the large number of chopstick manufacturers as well as uneven quality have resulted in low material utilization and aggravated the waste of natural resources. It is reported that there are thousands of domestic companies producing DCs, consuming nearly 5 million cubic meters of wood resources each year. During the process of producing chopsticks, i.e., from logs to wood blocks to finished products, the effective utilization rate of wood is 60\% ( $\mathrm{Li} \mathrm{2006).} \mathrm{In} \mathrm{addition,} \mathrm{most} \mathrm{of} \mathrm{the}$ waste DCs are buried as garbage, and have not yet begun to be recycled, which makes the situation even worse.

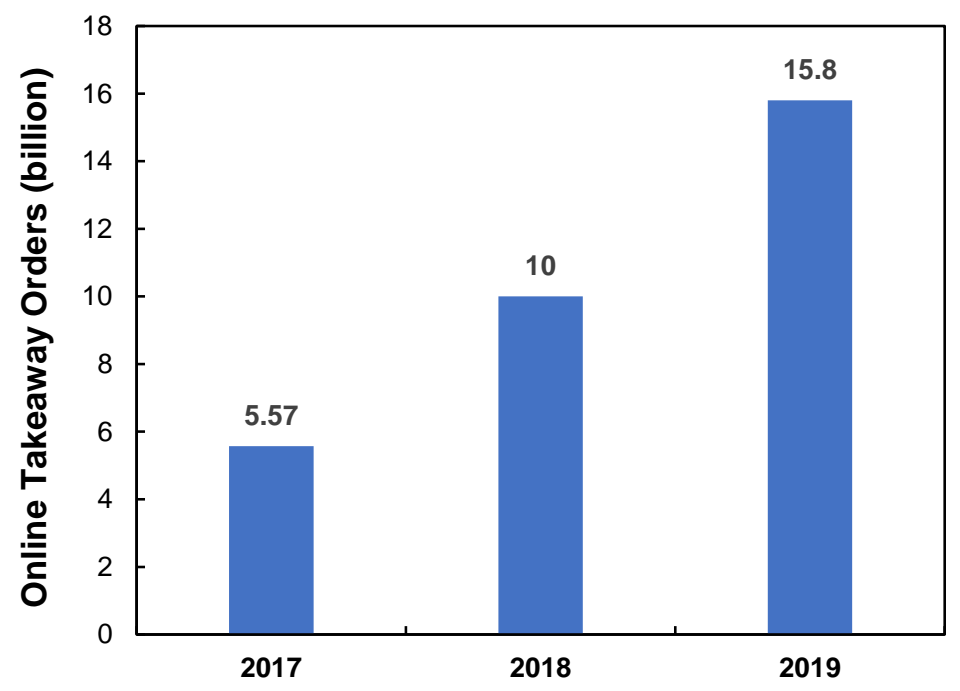

Fig. 1. The Annual online takeaway orders from 2017 to 2019

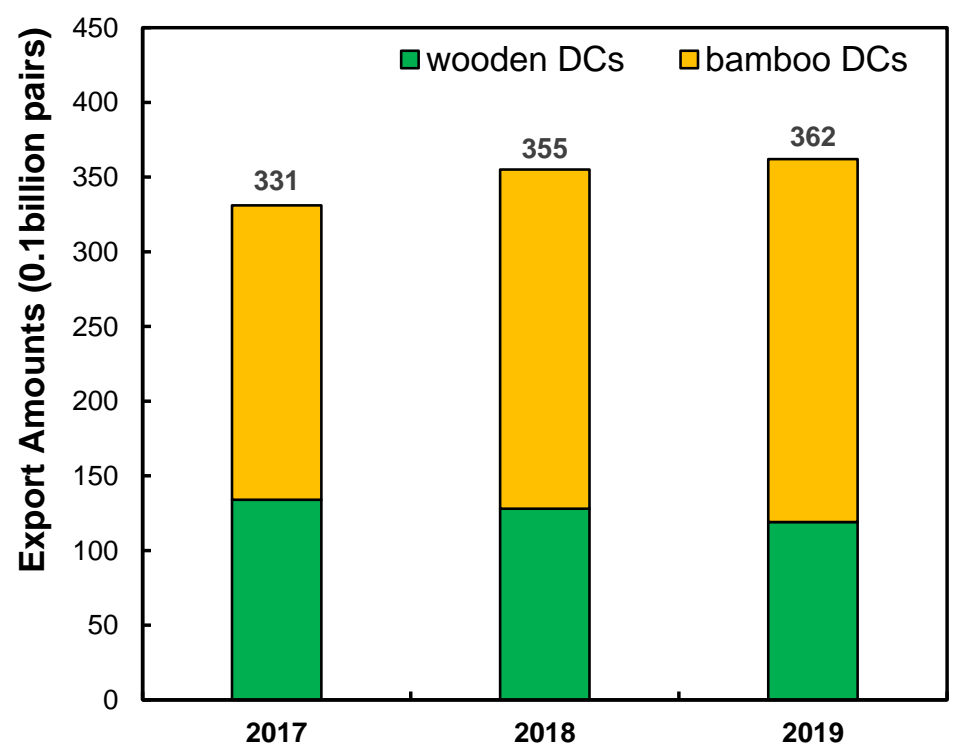

Fig. 2. The annual export volume of disposable chopsticks from 2017 to 2019

The negative effects of the production and disposal of DCs on the environment and human health have attracted increasing attention. A large number of takeaway orders resulted in $323 \mathrm{kt}$ of tableware and packaging waste in 2018, which is equal to three-fifths 
of the overall municipal solid waste generation of China per day (Marsh and Bugusu 2007; Dilkes-Hoffman et al. 2018). The online takeaway ordering industry in China produced large amounts of greenhouse gases $\left(709 \mathrm{kt}\right.$ of $\left.\mathrm{CO}_{2}\right)$, toxic gases $\left(\mathrm{SO}_{2}\right.$ and $\left.\mathrm{NOx}\right)$, and fine particulate matter (PM2.5). In addition, chopsticks are one of the primary contributors of $\mathrm{SO}_{2}$ emissions (45.59 kt), NOx emissions (333.65 t), PM2.5 emissions (23.55 t), and water emissions $\left(307570 \mathrm{~m}^{3}\right)$ (Zhou et al. 2020).

\section{Lack of Recycling Awareness}

Although Chinese residents have gradually realized the value of recycling, there is still a big gap between the environmental awareness of the Chinese people and that of developed countries. Most residents only pay attention to some common daily recyclable items, e.g., plastic bottles, cans, cardboard, etc., while other recyclable resources such as chopsticks tend to be ignored. During the initial stage of the implementation of garbage sorting and the removal of the trash bins in the community in July 2019 in Shanghai, many residents have not adapted to the habit of regularly throwing away garbage at fixed points and, some people still throw rubbish on the green belt at the risk of being fined (Qi 2019). For DCs, which are not mandatory to be recycled in China, few consumers or manufacturers realize its recycling value. Almost all food and beverage establishments see used DCs as trash. In general, it is hard to change the minds of people overnight, which is also one of the obstacles to the recycling.

\section{Immature Recycling Technology}

Immature recycling technology is another major factor restricting the recycling of wooden chopsticks. Recycling DCs into paper is a relatively common recycling method, since plant-derived DCs contain a large amount of cellulose, which is an important raw material for papermaking. The first step for utilizing DCs to make paper is cleaning and disinfection. Most of the discarded DCs are stained with oil and even bacteria. Cleaning these DWCs is the key to their reuse. Small and medium-sized paper mills have limited funds and outdated technical equipment. In order to save costs, paper mills will use cheap detergents to clean DCs, but cheap detergents contain a lot of phosphorus and even exceed standards. Moreover, small companies lack advanced sewage treatment systems. If they discharge their wastewater directly into rivers or it infiltrates into the soil without proper treatment, it will cause water or soil pollution, which means the loss outweighs the gain. In addition to cleaning, other processes also need to be optimized and improved to reduce costs and pollution, as well as reuse methods that are not limited to paper and fuel.

\section{Incomplete Recycling System}

China implemented garbage classification in 2019, and the construction of the recycling system started late, resulting in immature recycling systems. Although China produces approximately 50 billion pairs of DCs every year for the domestic market, chopstick waste is scattered across the country in towns, hotels, restaurants, and canteens of various sizes, which increases the difficulty of recycling. The consumption of DCs in small towns is insufficient for recycling, and it is difficult to form a batch processing scale; the number of DCs in hotels and restaurants in big cities is sufficient but too scattered. It is very difficult to gather these discarded DCs together in a short time. Even worse, the shelf life of DCs is 4 months at the longest, lasting only 3 months during the summer. If the recycled DCs cannot be quickly utilized and put into production, they will quickly pile up, spread germs, and even attract rodents and insect pests. Therefore, it is important to 
establish a scientific and reasonable recycling system that can collect scattered chopstick waste for centralized processing.

\section{Limitation Of Policy Effectiveness}

As more and more DCs are produced in China, the attitudes of the central and local governments toward DCs have gradually changed; taxation has shifted from export tax rebates to export taxation, and policies have gradually shifted from guiding and restricting the use to legislative prohibition. In order to limit the destruction of forest resources caused by the large export of DCs, the state also specifically abolished the $13 \%$ export tax rebate for disposable chopsticks in 2004. In November 2006, an additional 10\% export duty was imposed, during which 5\% of the product was restored (Yuan and Gao, 2007). A consumption tax and $17 \%$ value-added tax have also since been added. As early as 2001, Shanxi Province banned the production and use of DCs where forest resources were scarce. In the "Operating standards of catering enterprise" standard promulgated in 2007 (SB/T 10426-2007, 2007), the Ministry of Commerce first proposed the requirement of "reducing the use of DCs, paper cups and other tableware in the catering industry". However, these laws and regulations have had little effect, and the difficulty of implementation has become the biggest common problem.

\section{RESOLUTION STRATEGIES}

In order to handle the problems of wasting natural resources as well as the environmental issues caused by DCs, solution strategies are primarily introduced from two aspects: the green design of DCs and the recycling of waste chopsticks. Green design focuses on the root of the problem and aims to design and manufacture environmentally friendly DCs. The construction of a recycling system plays an indispensable role in the recycling of chopstick waste, and several campus and social recycling systems have been proposed. Since the chopsticks are based on wood or bamboo, different approaches to turn waste into wealth are introduced (Chen et al. 2021), including but not limited to yielding chemical raw materials, new materials, energy, furniture, etc. An overview of the resolution strategies is shown in Fig. 3.

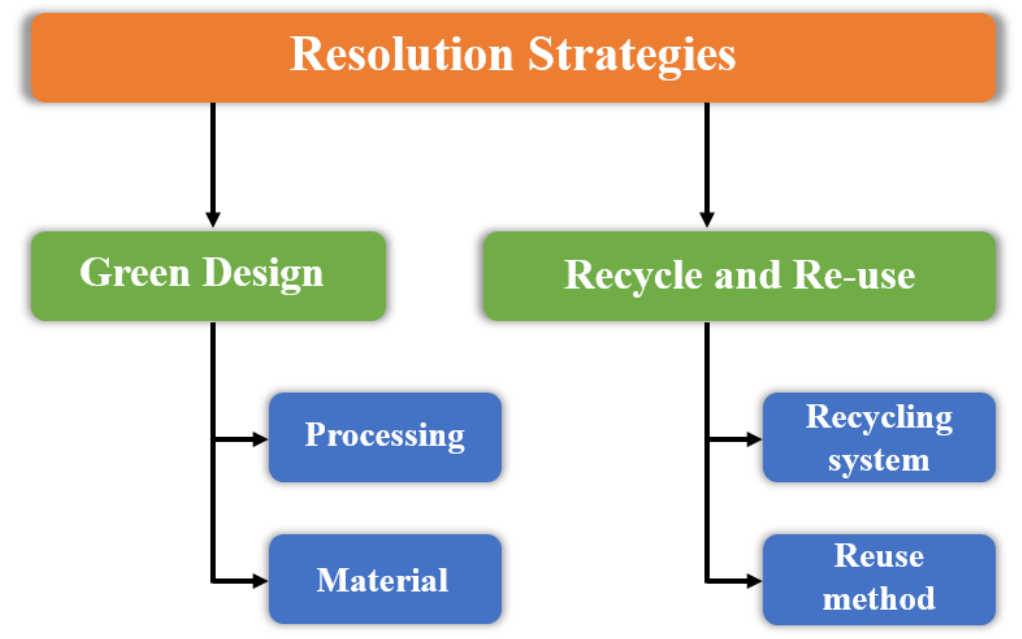

Fig. 3. Overview of the resolution strategies 


\section{Green Design Methods for Disposable Chopsticks}

Optimization of the industrial production process

Optimizing the traditional production process can effectively reduce the energy consumption and production costs of the process, and some special optimized production methods can improve the quality of the chopsticks, thus increasing their life cycle. Yu (1995) discussed the drying process in order to enhance the quality of poplar chopsticks. During the drying process, Yu (1995) found that the drying oven should not be overfilled, because excessive accumulation will not only make the chopsticks at the bottom of the oven easily bent, but also affects the ventilation and reduces the drying speed. In addition, the temperature during the drying process needs to be reasonably controlled. During the initial drying stage, excessively high temperatures will not only restrain the removal of water, making the chopsticks dirty, but also waste energy. In order to prevent the deformation of wooden chopsticks, the heating speed should not be too fast, so they used a self-made drying oven and increased the temperature at a speed of $10{ }^{\circ} \mathrm{C} / \mathrm{h}$ to $15^{\circ} \mathrm{C} / \mathrm{h}$. The chopsticks dried by this method had a small amount of deformation (the front warpage did not exceed $3 \mathrm{~mm}$ and the side warpage did not exceed $1.5 \mathrm{~mm}$ ). Li (2011) proposed an antibacterial processing method for wooden or bamboo tableware. First, the chopsticks are put into an oven and baked at a high temperature of 200 to $300{ }^{\circ} \mathrm{C}$ for 10 to 120 minutes. Second, the chopsticks were taken out and immediately immersed in cold water so as to form a very thin carbon crystal layer on the surface (Yang et al. 2021), which has the advantages of a high hardness and are non-sticking, antibacterial, etc.

\section{Material substitution}

In order to alleviate the predicament of scarce timber resources, seeking a low cost, renewable, and biodegradable alternative material has become the development trend of future chopsticks. One of the most commonly used materials for DCs is bamboo (as shown in Fig. 4a), which has the advantages of lighter mass, smooth surface, regular texture, antibacterial properties, etc. (Chen et al. 2017). Besides bamboo, other biomass materials have also become intensively pursued candidates. Li (2002) proposed all-waste-derived DCs made of straw and waste paper. The chopstick core was composed of waste straw powder from various crops, while the waste paper was wrapped around the chopsticks to form an enhanced layer. The novel chopsticks were completely based on waste and could alleviate the massive consumption of bamboo and wood resources. Analogously, Lian (2018) reported a new kind of DCs made from rice hulls. The ferulic acid in the rice husks is a natural preservative, which is effectively antibacterial and does not harm to the health of the users. In addition, the surface smooth level, mechanical property and microbial content of the prepared DCs were tested to meet the standards of commercial chopsticks. Zhou (2007) invented fascinating silicone composite chopsticks consisting of a chopstick core and a glue layer. The grip and front end of the wooden or bamboo chopstick core were covered by a silicone layer, which served as a protective layer for the core and prolonged its service life. In addition, the silica gel provided users with a more comfortable experience due to its inherent softness.

Thanks to the novel idea of making chopsticks a dessert after dinner, edible chopsticks have attracted the attention of people in recent years. Marushige Confectionery, a Tokyo-based company, released new edible chopsticks with a "Tatami" flavor (Casey 2017; Clara 2017). Instead of being formed out of wood, metal, or other common materials, these chopsticks are made from igusa, a type of reed that is typically used in the production of traditional Japanese tatami floor mats (as shown in Fig. 4b). The "tatami-flavored" 
chopsticks are not delicious, but they bring to mind a bitter, dried grass taste; however, they contain abundant dietary fiber. Surprisingly, the original intention of the chopsticks was not motivated by ecological concerns, but to preserve a part of Japanese agricultural heritage. However, Marushige does deserve credit for coming up with a creative way to help the environment. In contrast, the leading Chinese food delivery platform Eleme (Esgn 2019) produces tasty edible chopsticks with three flavors, including matcha flavor, wheat flavor, and purple sweet potato flavor (as shown in Fig. 4c). The primary ingredients in these chopsticks are not wood, but wheat flour, butter, icing sugar, milk, and water. Even if they are not eaten, they will naturally degrade within a week.
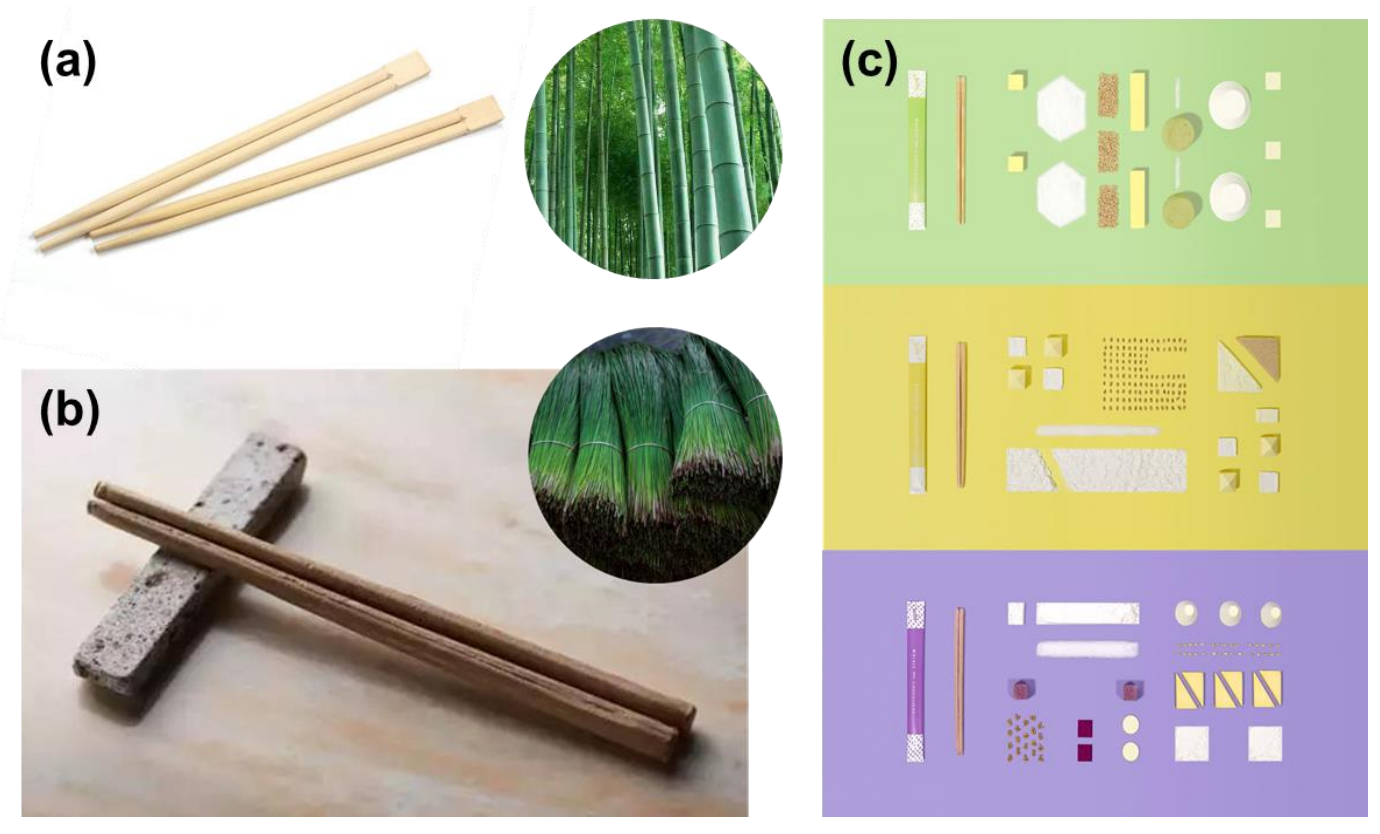

Fig. 4. The disposable bamboo chopsticks (a); the "tatami-flavored" edible chopsticks made from igusa (b); and the edible chopsticks with three flavors from Eleme (c)

\section{Recycling Strategies of Waste Chopsticks}

DCs, as well as the chopstick waste, belong to the category of lignocellulosic materials, which are the most abundant and available biodegradable resources on Earth (Zuin et al. 2018). Nowadays, a variety of technologies are available for the conversion of biomass or bio-waste to different energy forms including biochemical, thermal chemical, and physical processes (Qiu and $\mathrm{Hu} 2013$; Fieschi and Pretato 2018). Energy derived from biomass and biological waste can be in solid, liquid, or gaseous forms.

Chen et al. (2017) processed a solid bio-fuel based on waste chopsticks via torrefaction using a tubular furnace with nitrogen as the carrier gas (the schematic diagram is shown in Fig. 5a). The obtained fuel not only met the standards for industrial fuels, but also was easy to store, transport, and process due to the hydrophobic surface modification. In comparison with solid and gas fuels, liquid fuels possess more advantages, e.g., convenient storage, easy transportation, and a wide range of uses. Chang et al. (2016) employed a $\mathrm{K}_{2} \mathrm{CO}_{3}$ catalyst for hydrothermal liquefaction (HTL) to convert the biomass of waste chopsticks into bio-oil. The results revealed that $\mathrm{K}_{2} \mathrm{CO}_{3}$ was an effective catalyst for the HTL of waste chopstick biomass with considerable lignin content (up to $25.5 \mathrm{wt}$.\% in dry-basis). In addition, the bio-oil has similar simulated distillation characteristics to diesel, which is better than heavy oil and boat oil. 
(a)

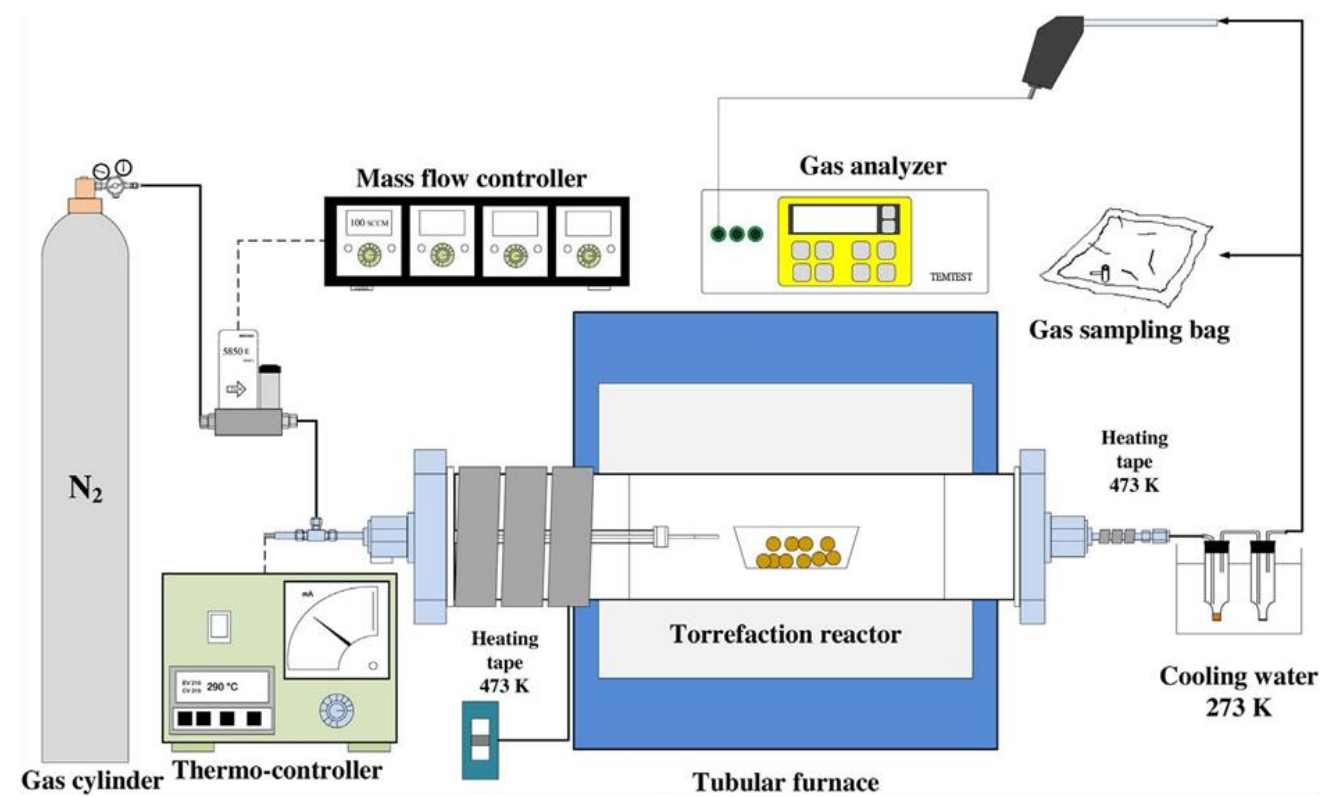

(b)
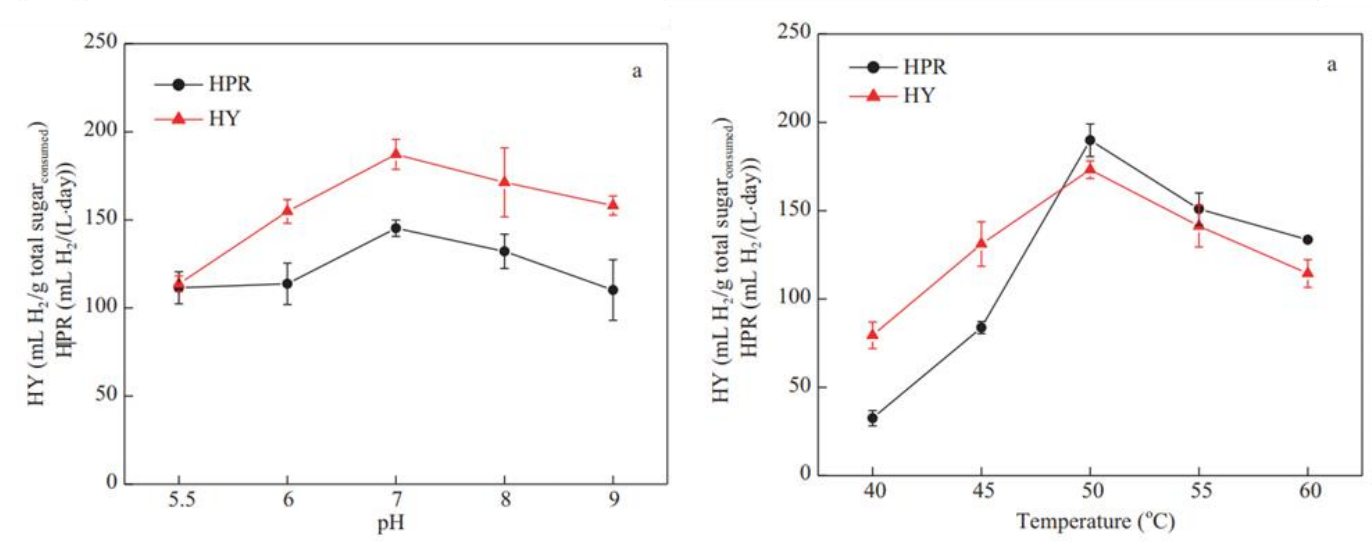

Fig. 5. The schematic diagram of the torrefaction system (Chen et al. 2017) (a); the influence of the initial $\mathrm{pH}$ and temperature on hydrogen production (HPR: $\mathrm{H}_{2}$ production rate; $\mathrm{HY}: \mathrm{H}_{2}$ yield) (Phummala et al. 2014) (b); Reprinted with permission by Elsevier

Phummala et al. (2014) demonstrated the feasibility of using DWCs as a feedstock for fermentative $\mathrm{H}_{2}$ and the optimal conditions for the enriched hot spring culture. The results showed that an initial $\mathrm{pH}$ of 7.0 in the culture medium incubated at a temperature of $50{ }^{\circ} \mathrm{C}$ attained the highest $\mathrm{H}_{2}$ production (Fig. 5b). Asada et al. (2011) produced bioethanol from disposable aspen chopsticks via ball milling, steam explosion, enzymatic saccharification, and simultaneous saccharification and fermentation. During the enzymatic saccharification of ball-milled chopsticks, the largest amount of glucose was obtained after $60 \mathrm{~min}$ of ball-milling, which was $520 \mathrm{mg}$ of glucose per $\mathrm{g}$ of dry aspen chopsticks. Shih et al. (2012) prepared novel fiber-reinforced green composites by adding modified DCs fibers and polypropylene-graft-maleic anhydride to polypropylene (PP). Recycled DCs fibers were incorporated into the PP to improve the mechanical and thermal properties of the plastics. The results showed that the obtained composites exhibited excellent characteristics, and the maximum increments of the tensile were $66 \%$ and the heat-deflection temperature reached up to $144.8^{\circ} \mathrm{C}$. 
Value-added designs will be the focus of waste recycling in the future (Xiong et al. 2020). Some companies have devised methods to upcycle waste chopsticks into furniture and interior products to give them a second life cycle (Carlson and Rafinejad 2011; Xiong et al. 2017). The Canadian startup Chop Value places recycling bins at participating restaurants in Vancouver and collects the chopsticks, giving them a second life cycle as interior products. By recycling the chopsticks, restaurants can save money on garbage collection costs, while also improving their environmental footprint. The bamboo chopsticks are processed into a new material, which still has the characteristics of bamboo. The sticks are first cleaned, then coated in resin, and finally hot-pressed into a laminated material (Xiong et al. 2020). The "planks" are cut and put together into a range of interior products, e.g., wall tiles, yoga blocks, and coasters (Fig. 6a) (Sun et al. 2019). They also produce tabletops made from chopsticks. The base is handcrafted out of reclaimed wood or salvaged steel sourced from local, demolished construction sites. Of all the products, the most prominent is the SMİLE shelf, a modular system that relies on steel frames fixed to the wall to clip the shelves in between (Sheth 2020). Chop Value gathers DCs waste and turns them into new sturdy material that can be used again. Each shelf is composed of hundreds of bamboo chopsticks, compressed together to obtain a unique looking material (Fig. 6b).

It is inspiring that everyone could use discarded old chopsticks to create a lot of imaginative things in our daily lives. Rinkesh (2021) proposed that abandoned DCs can be given a new beginning by being employed as stirrers, pegs, corner cleaners, hair bun holders, etc. Trish (2012) listed several innovative and extraordinary ways to recycle wooden chopsticks, e.g., making recycled chopstick into folding basket, lamps, dress, handbag, wine bottle holder, etc. For individuals, although they may not be able to eliminate DCs' negative effects immediately, they can do better by limiting DCs' impact on the environment.

(a)

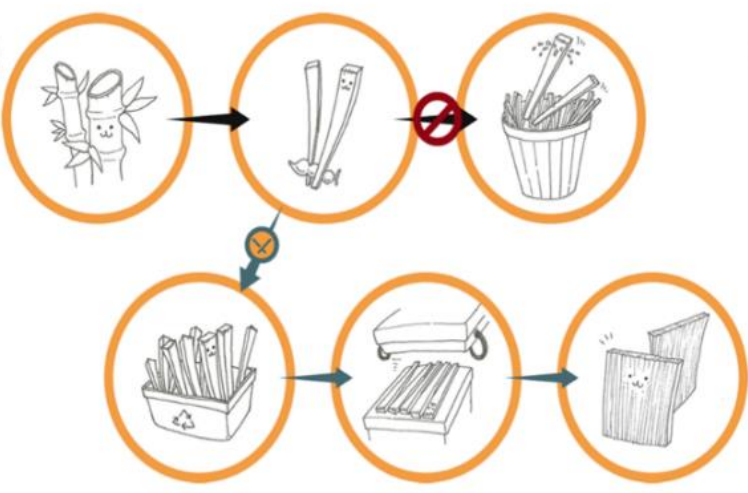

(b)

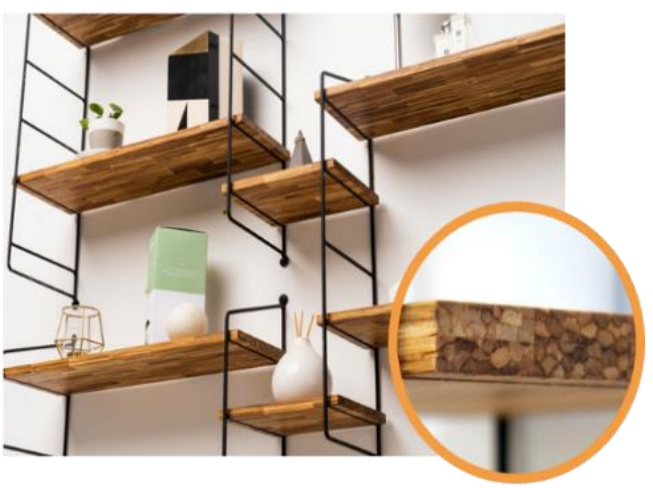

Fig. 6. The schematic diagram of processing chopsticks waste into new materials by Chop Value (a); and the SMĪLE shelf and its unique cross-sectional pattern (b)

\section{Recycling System}

In recent years, many researchers have devoted themselves to the study of campus recycling systems, so as to establish recycling systems that can be applied to society and even the country. They evaluate the systems through simulation modeling and quantitative calculations and have made considerable headway. Yu (2018) pointed out the primary characteristics of campus waste: when eating in the canteen, consumers have developed 
the habit of actively participating in the sorting work, resulting in a high recycling efficiency; when consumers choose to eat in the dormitory, the recovery rate is low. However, the primary participants in dormitory waste sorting are cleaners rather than the students, which causes waste sorting to lag behind its generation. Yu (2018) proposed the campus waste recycling process (as shown in Fig. 7) and used quantitative calculations to determine the location of the recycling points and the recycling center. Chen (2016) extended the campus recycling system to the social system and simulated a reverse logistics network for DCs. The initial collection points were established in concentrated areas, e.g., hotels, restaurants, canteens, and snack streets, which would allow the chopsticks to be recycled regularly and be sent to the recycling station. The recycling center was responsible for recycling the waste at the recycling points in the area, and sorting them, distinguishing DCs that can be reused. The processing center was responsible for reprocessing the recycled DCs. Recycled disposable chopsticks were cleaned and cut into pulp, which was used as a raw material for the paper mill, while those that could not be recycled were packed and sent to fuel plants. By reprocessing, paper mills and fuel mills turned disposable chopsticks into new products, which benefited consumers.

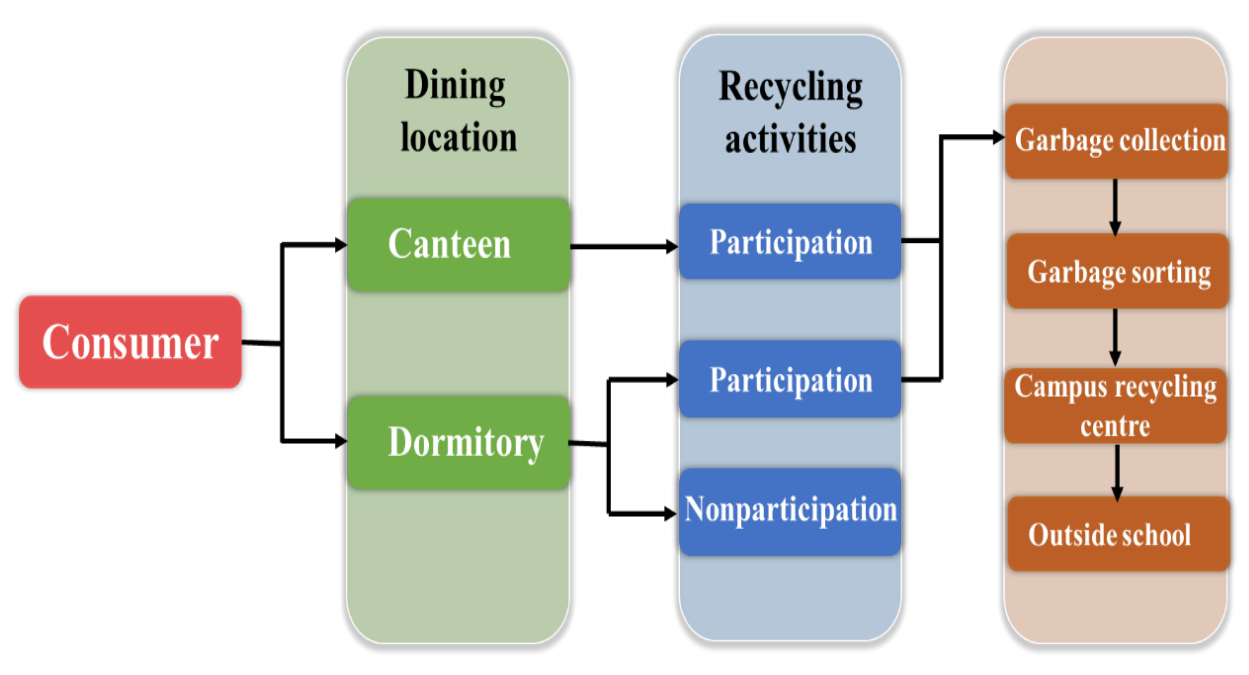

Fig. 7. The campus DCs waste recycling process

\section{DISCUSSION}

So far, various methods have been proposed to manufacture chopstick waste into products, ranging from industrial energy resources to furniture used in daily life. Enterprises, scientific researchers, and even individuals actively offer advice and suggestions for the reuse of chopstick waste. As is known to all, garbage classification and collection are important foundations for the recycling of chopstick wastes (Kurniawan $e t$ al. 2021). However, the garbage classification policy in China was implemented late, so it is indispensable to learn from the developed recycling systems of other regions (Chen $e t$ al. 2020; Tong et al. 2020). Based on mature experiences and lessons learned over the past decades, waste management challenges in China in the near future can be foreseen, prevented, and addressed. 


\section{Proposed Methods for Construction of DCs Recycling System}

The establishment of a recycling system cannot be achieved without the cooperation of manufacturers, consumers, and the government. As learned from South Korea and Taiwan, two policies aimed at manufacturers and consumers are effective for garbage recycling (Houng et al. 2014; Min and Rhee 2014). One is called the "Waste Charge System", which charges the manufacturers and importers the cost involved in the disposal of a product that is not eco-friendly or is difficult to be recycled. This system aims at reducing the waste generated at the manufacturing stage and promotes efficient waste disposal by taking into account the possibility of the environmental impacts caused by products. Another system used to restrict users is the "User Pay System", which has the intention of reducing the waste during the production stage. This system forces people to pay a disposal fee based on the amount of waste they produce (Min and Rhee 2014). These two recycling principles can be applied to the treatment of chopstick wastes through the regulation of government policies. The government should regulate and classify the materials of DCs. Chopstick manufacturers using fast-growing wood and bamboo to produce chopsticks are considered environmentally friendly, and they either do not need to pay or should pay less for recycling, whereas chopsticks made from expensive wood means that the manufacturers should take more responsibility. For takeaway platforms, they should provide chopsticks for a fee according to the needs of the customers, instead of providing them completely for free, which is intended to guide people to form a frugal lifestyle and reshape their consumption concept. Facing the explosive consumption of DCs, a number of initiatives in China have sought similar solutions, including three nonbinding food packaging standards by Shanghai Association of Food Contact Materials in June 2018 and a single-use tableware ban in Beijing in May 2020 and in Shenzhen in September 2020. Although these policies have effectively reduced the use of disposable tableware, the implementation of policies still has a long way to go, since the popularization rate of the policies is not high, and they are only implemented in some developed cities. The "User Pay System" requires those who benefit from the use of a resource to pay costs associated with the loss of the resource, as well as the full costs for use of the resource and the services associated with it. From the perspective of the customer, they can do a bit to protect the environment by choosing the "no tableware" service when ordering takeaway food.

In the past, the use of DCs was concentrated in restaurants, canteens, and for eating takeaway lunches bought at convenience stores and supermarkets. However, the emergence of food delivery has changed this situation and hindered the recycling of chopsticks. Due to the rapid development of the food delivery industry, it is now possible to enjoy the convenience of food delivery with disposable tableware by ordering takeaway anytime and anywhere; this makes the distribution of chopstick wastes wide and scattered, increasing the difficulty of collection. This requires the entirety of people to increase their awareness in terms of recycling and actively participate in garbage sorting after using disposable tableware. However, Rome was not built in a day, and garbage sorting in China has just been implemented, and proper recycling habits have not yet been developed, so it is necessary to implement a reward system. In general, the reward system is usually more effective than the punishment system. While the "User Pay System" encourages users to bring their own chopsticks instead of using disposable chopsticks by charging, when consumers have to use disposable tableware, they can recycle them and benefit from it, according to the reward system. Specifically speaking, if a person recycles disposable tableware after finishing takeaway food, they can get a red envelope subsidy provided by a takeaway platform, which is a win-win strategy for the government, delivery platforms, 
and consumers (Fig. 8). Through the establishment of a "recycling convenience store" by the government, an incentive policy can also be applied to the recycling of community waste. As of January 2021, Hong Kong has set up 18 "recycling convenience stores", where citizens can earn points by recycling specific waste to redeem daily food and household items, stimulating the enthusiasm of people for participating in recycling. It also implemented a reward system for reporting the unlawful use of disposable products, to establish the restriction system quickly through the participation of citizens. Restaurants can establish tableware collection points through cooperation with enterprises and the government, so as to obtain government subsidies while achieving good economic benefits.

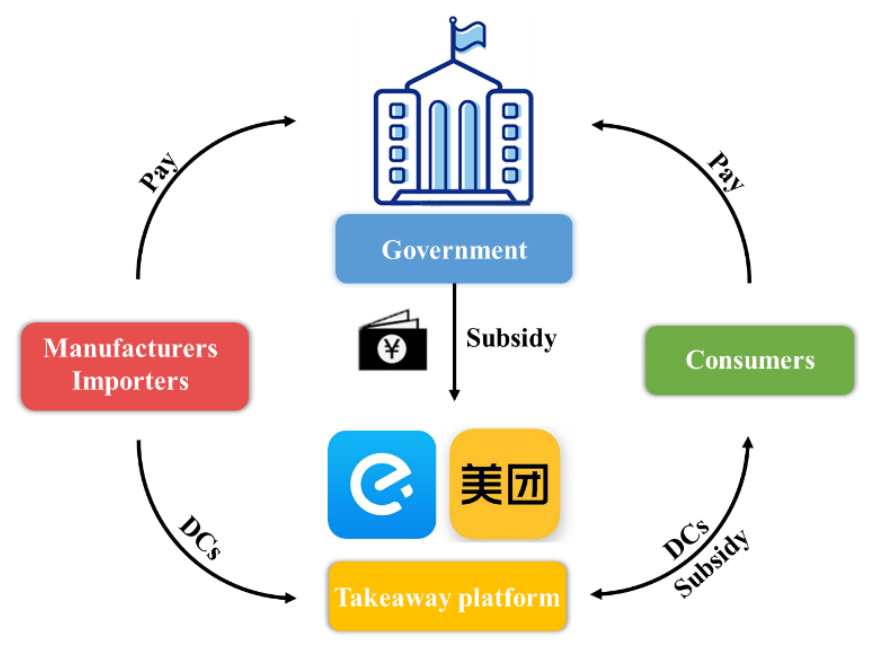

Fig. 8. Operation of the recycling system and the reward/punishment system

\section{CONCLUDING STATEMENTS}

In this review, the authors summarized the difficulties in recycling disposable chopsticks (DCs) and reported a series of solutions to these problems. It is shown that the resolution strategies are primarily based on green designs and the recycling of waste chopsticks, so as to produce green chopsticks or enable them a second life cycle as other products. The immature garbage collection system in China has become the biggest obstacle to the recycling of DCs, so several practical recycling systems based on government experience in developed regions, tailored to China's national conditions, were proposed.

1. Seeking a low-cost, renewable, and biodegradable alternative material can fundamentally alleviate the shortage of wood resources. Besides bamboo, several biomass materials are considered as potential materials for manufacturing DCs, e.g., straw, wheat, rice hull, waste paper, etc. To transition toward a more sustainable society based on a circular economy, bio-waste will become the most promising alternative in future research.

2. As China lacks a complete garbage collection system, it is helpful for the government to formulate reasonable policies by drawing lessons from developed regions that have effective polices for dealing with the same problems. The principles of "users pay" 
and "polluters pay" have been shown to effectively reduce waste from the production stage by charging fees according to the quantity of waste produced.

3. It can be foreseen that various reward policies will be implemented in the near future, such as 'Recycling Convenience Stores' and 'Takeaway subsidies', which has a good influence on stimulating the enthusiasm of individuals for recycling disposable tableware.

4. The location of recycling points still requires a large amount of data for testing and simulation, as unreasonable collection points may cause negative effects. Due to the large number of restaurants in China, it is unrealistic to set up recycling points in each restaurant. However, even if multiple restaurants share the same recycling point, the new recycling points still increase the workload and cost of garbage collection, i.e., the newly added recycling line increases the fuel consumption of the garbage collection truck. This leads to an increase in the emission of air pollutants, e.g., NOx and particulates.

\section{ACKNOWLEDGMENTS}

The authors are grateful the support of the Joint Research program of the Sinoforeign Cooperation in Running Schools of Jiangsu Province, China.

\section{REFERENCES CITED}

Asada, C., Kita, A., Sasaki, C., and Nakamura, Y. (2011). "Ethanol production from disposable aspen chopsticks using delignification pretreatments," Carbohydrate Polymers 85(1), 196-200. DOI: 10.1016/j.carbpol.2011.02.020

Blanca-Alcubilla, G., Bala, A., Castro, N. d., Colomé, R., and Fullana-i-Palmer, P. (2020). "Is the reusable tableware the best option? Analysis of the aviation catering sector with a life cycle approach," Science of The Total Environment 708, 1-8. DOI: 10.1016/j.scitotenv.2019.135121

Carlson, R. C., and Rafinejad, D. (2011). "The transition to sustainable product development and manufacturing," in: Planning Production and Inventories in the Extended Enterprise: A State of the Art Handbook, Volume 1, K. G. Kempf, P. Keskinocak, and R. Uzsoy (ed.), Springer, New York, NY.

Casey, B. (2017). "Japan's new edible chopsticks have a special flavor, but don't taste like 'food," SoraNews24, (https://soranews24.com/2017/04/04/japans-new-ediblechopsticks-have-a-special-flavor-but-dont-taste-like-food/), accessed 29 March 2021.

Chang, C.-C., Chen, C. -P., Yang, C. -S., Chen, Y. -H., Huang, M., Chang, C. -Y., Shie, J. -L., Yuan, M. -H., Chen, Y. -H., Ho, C., et al. (2016). "Conversion of waste bamboo chopsticks to bio-oil via catalytic hydrothermal liquefaction using $\mathrm{K}_{2} \mathrm{CO}_{3}$," Sustainable Environment Research 26(6), 262-267. DOI: 10.1016/j.serj.2016.08.002

Chen, B. Z. (2016). Research on Logistics Model and Network of Disposable Chopsticks, Master's Thesis, Jiangxi University of Science and Technology, Jiangxi, China.

Chen, H., Yu, Y., Zhong, T., Wu, Y., Li, Y., Wu, Z., and Fei, B. (2017). "Effect of alkali treatment on microstructure and mechanical properties of individual bamboo fibers," Cellulose 24(1), 333-347. DOI: 10.1007/s10570-016-1116-6 
Chen, Y.-H., Chang, C. -C., Chang, C. -Y., Yuan, M. -H., Ji, D. -R., Shie, J. -L., Lee, C.H., Chen, Y. -H., Chang, W. -R., Yang, T. -Y., et al. (2017). "Production of a solid bio-fuel from waste bamboo chopsticks by torrefaction for cofiring with coal," Journal of Analytical and Applied Pyrolysis 126, 315-322. DOI: 10.1016/j.jaap.2017.05.015

Chen, S., Huang, J., Xiao, T., Gao, J., Bai, J., Luo, W., and Dong, B. (2020). “Carbon emissions under different domestic waste treatment modes induced by garbage classification: Case study in pilot communities in Shanghai, China," Science of The Total Environment 717, 1-12. DOI: 10.1016/j.scitotenv.2020.137193

Chen, H., Wu, J. Y., Shi, J. J., Zhang, W. F., and Wang, H. K. (2021). "Effect of alkali treatment on microstructure and thermal stability of parenchyma cell compared with bamboo fiber," Industrial Crops and Products 164, article no. 113380. DOI: 10.1016/j.indcrop.2021.113380

China Cuisine Association. (2018). China's Catering Industry Development Report 2018, China, Beijing.

Clara, O. (2017). "Could edible chopsticks save Japan's Agriculture?," FOOD\&WINE, (https://www.foodandwine.com/news/could-edible-chopsticks-save-japansagriculture), accessed 4 July 2021.

Dilkes-Hoffman, L. S., Lane, J. L., Grant, T., Pratt, S., Lant, P. A., and Laycock, B. (2018). "Environmental impact of biodegradable food packaging when considering food waste," Journal of Cleaner Production 180, 325-334. DOI: 10.1016/j.jclepro.2018.01.169

Esgn Asia (2019). "No need to waste food. You can eat your chopsticks now!," ESG Newswire, (https://esgn.asia/no-need-to-waste-food-you-can-eat-your-chopsticksnow/), accessed 29 March 2021.

European Environment Agency (2016). Circular Economy in Europe: Developing the Knowledge Base (EEA Report No. 2/2016), Publications Office, European Union.

Faraca, G., Boldrin, A., and Astrup, T. (2019). "Resource quality of wood waste: The importance of physical and chemical impurities in wood waste for recycling," Waste Management 87, 135-147. DOI: 10.1016/j.wasman.2019.02.005

Fieschi, M., and Pretato, U. (2018). "Role of compostable tableware in food service and waste management. A life cycle assessment study," Waste Management 73, 14-25. DOI: $10.1016 /$ j.wasman.2017.11.036

General Administration of Customs. (2020). Summary of Disposable Chopsticks Exports, China, Beijing.

Houng, H., Shen, S.-H., and Ma, H.-K. (2014). "Municipal solid waste management in Taiwan: From solid waste to sustainable material management," in: Municipal Solid Waste Management in Asia and the Pacific Islands: Challenges and Strategic Solutions, A. Pariatamby and M. Tanaka (ed.), Springer, Singapore.

Ju, F. C. J. (2021). "Half a billion people "contributed"! With more than 17.1 billion takeout orders a year, which two groups contribute the most?," Baidu, (https://baijiahao.baidu.com/s?id=1687761109814871039\&wfr=spider\&for=pc), accessed 4 October 2021

Kissen. (2017). "Some thoughts on the import and export trade of disposable chopsticks in China," Flavours, (https://www.pinlue.com/article/2017/10/0921/514658939140.html), accessed 2 October 2021. 
Kurniawan, T. A., Lo, W., Singh, D., Othman, M. H. D., Avtar, R., Hwang, G. H., Albadarin, A. B., Kern, A. O., and Shirazian, S. (2021). "A societal transition of MSW management in Xiamen (China) toward a circular economy through integrated waste recycling and technological digitization," Environmental Pollution 277, 1-18. DOI: $10.1016 /$ j.envpol.2021.116741

Li, J. P. (2002). "Disposable environmentally friendly chopsticks," CN Patent No. 01252634.7.

Li, M. (2018). "Market development overview of China's disposable chopsticks industry in 2018,"Souhu, (https://www.sohu.com/a/www.sohu.com/a/225595569_252291), accessed 30 September 2021.

Li, P. (2006). "Disposable chopsticks cause right and wrong," Ecological Economy 2(6), 14-19.

Li, W. Q. (2011). “An antibacterial processing method for wooden or bamboo tableware," CN Patent No. 200910108424.9.

Lian, B. L. (2018). "Study on the production technology of disposable rice husk chopsticks," Forestry Science \& Technology 43(02), 54-56.

Liu, Q. Q., Gao, D., and Xu, W. (2020). "Effect of sanding processes on the surface properties of modified poplar coated by primer compared with mahogany," Coatings 10, 856. DOI: 10.3390/coatings 10090856

Marsh, K., and Bugusu, B. (2007). "Food packaging - Roles, materials, and environmental issues," Journal of Food Science 72(3), R39-55. DOI: 10.1111/j.17503841.2007.00301.x

Meituan Research Institute. (2018). Research Report on China Takeaway Development in 2017, China.

Min, D.-K., and Rhee, S.-W. (2014). "Management of municipal solid waste in Korea," in: Municipal Solid Waste Management in Asia and the Pacific Island s: Challenges and Strategic Solutions, A. Pariatamby and M. Tanaka (eds.), Springer, Singapore.

Molina-Besch, K. (2020). "Food delivery packaging and tableware waste," Nature Food 1(9), 531-532. DOI: 10.1038/s43016-020-00146-Z

Osens. (2021). "Osens takes you a comprehensive perspective on the scale, development trend and competitive landscape of China's chopsticks market," Zhihu, (https://zhuanlan.zhihu.com/p/365703676), accessed 18 May 2021.

Phummala, K., Imai, T., Reungsang, A., Chairattanamanokorn, P., Sekine, M., Higuchi, T., Yamamoto, K., and Kanno, A. (2014). "Delignification of disposable wooden chopsticks waste for fermentative hydrogen production by an enriched culture from a hot spring," Journal of Environmental Sciences 26(6), 1361-1368. DOI: 10.1016/S1001-0742(13)60612-9

Qi, Y. P. (2019). "People who litter will be fined up to the top of the list, according to new rules for Shanghai municipal waste management," Souhu, (https://www.sohu.com/a/421528703_120214184), accessed 30 July 2021.

Qiu, X., and Hu, S. (2013). "'Smart' materials based on cellulose: A review of the preparations, properties, and applications," Materials 6(3), 738-781. DOI: $10.3390 / \mathrm{ma} 6030738$

Rinkesh. (2021). “Are Chopsticks Recyclable?," Paperblog, (https://en.paperblog.com/are-chopsticks-recyclable-and-are-they-compostable-orbiodegradable-2685989/), accessed 29 September 2021.

SB/T 10426-2007 (2007). "Operating standards of catering enterprise," Ministry of Commerce of the People's Republic of China, Beijing, China. 
Sheth, S. (2020). "This modular shelf is made from more than 4,000 recycled bamboo chopsticks!," YANKO DESIGN, (https://www.yankodesign.com/2020/09/21/thismodular-shelf-is-made-from-more-than-4000-recycled-bamboo-chopsticks/), accessed 30 March 2021.

Shih, Y.-F., Chen, P.-W., Wu, C.-S., Huang, C.-M., and Hsieh, C.-F. (2012). "Recycleddisposable-chopstick-fiber-reinforced polypropylene green composites," Journal of Applied Polymer Science 123(5), 3046-3053. DOI: 10.1002/app.34857

Sun, S., Zhao, Z., Shen, J. (2019). "Effects of the manufacturing conditions on the VOCs emissions of particleboard," BioResources 15(1), 1074-1084. DOI: 10.15376/biores.15.1.1074-1084

Tong, Y., Liu, J., and Liu, S. (2020). "China is implementing 'garbage classification' action," Environmental Pollution 259, 1-2. DOI: 10.1016/j.envpol.2019.113707

Trish, S. (2012). "10 Innovative and funky ways to recycle wooden chopsticks," Recyclebank, (https://livegreen.recyclebank.com/10-innovative-and-funky-waysrecycle-wooden-chopsticks), accessed 25 September 2021.

Xiong, X. Q., Ma, Q. R., and Ren, J. (2020). "The performance optimization of oriented strand board veneer technology," Coatings 10, 211. DOI: 10.3390/coatings 10060511

Xiong, X. Q., Ma, Q. R., Yuan, Y.Y., Wu, Z. H., and Zhang, M. (2020). “Current situation and key manufacturing considerations of green furniture in China A review," Journal of Cleaner Production 267, 121957. DOI:

10.1016/j.jclepro.2020.121957

Xiong, X. Q., Guo, W.J., Fang, L., Zhang, M., Wu, Z. H., Lu, R., and Miyakoshi, T. (2017). "Current state and development trend of Chinese furniture industry," Journal of Wood Science 63(5), 433-444. DOI: 10.1007/s10086-017-1643-2

Xu, R., Deng, S., Wang, W., Schenk, J., and Wang, F. (2020). "Structural features and combustion behaviour of waste bamboo chopstick chars pyrolysed at different temperatures," BioEnergy Research 13(2), 439-451. DOI: 10.1007/s12155-02010095-X

Yang, L. C., Wu, Y., Yang, F., and Wang, W. H. (2021). "The effect of antibacterial and waterproof coating prepared from hexadecyltrimethoxysilane and nano-titanium dioxide on wood properties," Frontiers in Materials 8, 261. DOI: 10.3389/fmats.2021.699579

$\mathrm{Yu}$, S. M. (1995). "Discussion on the process of steaming and drying of poplar sanitary chopsticks," China Forest Products Industry 5(2), 11-12.

Yu, Z. Y. (2018). Study on Campus Food Waste and Plastic Disposable Tableware Recycling System, Master's Thesis, Beijing University of Posts and Telecommunications, Beijing, China.

Yuan, Y., and Gao, Q. (2007). "Disposable chopsticks challenge China's national conditions.," IFENG, (https://news.ifeng.com/opinion/200709/ 0910_23_220334.shtml), accessed 2 August 2021.

Zhongshang Industry Research Institute. (2018). 2018-2023 China's Disposable Chopsticks Market Survey and Prospect Report, China, Shenzhen.

Zhou, Y., Shan, Y., Guan, D., Liang, X., Cai, Y., Liu, J., Xie, W., Xue, J., Ma, Z., and Yang, Z. (2020). "Sharing tableware reduces waste generation, emissions and water consumption in China's takeaway packaging waste dilemma," Nature Food 1(9), 552-561. DOI: 10.1038/s43016-020-00145-0

Zhou, Z. D. (2007). “Silica gel combined chopsticks,” CN Patent No. 200620013665. 
Zuin, V. G., Segatto, M. L., and Ramin, L. Z. (2018). "Plants as resources for organic molecules: Facing the green and sustainable future today," Current Opinion in Green and Sustainable Chemistry 9, 1-7. DOI: 10.1016/j.cogsc.2017.10.001

Article submitted: August 2, 2021; Peer review completed: September 28, 2021; Revised version received and accepted: October 14, 2021; Published: October 21, 2021.

DOI: $10.15376 /$ biores.16.4.Fu 